\title{
Fluid Enquiry, Complex Causality, Policy Processes: Making a Difference with Qualitative Longitudinal Research
}

\section{Bren Neale}

School of Sociology and Social Policy, University of Leeds

E-mail: b.neale@leeds.ac.uk

In this article, qualitative longitudinal (QL) research is presented as a powerful form of fluid enquiry. The combination of its real-world and real-time features enables this methodology to 'follow reality in all its windings', and, thereby, to produce useful knowledge for policy and professional practice. The main focus is on the power of QL methodology to illuminate complex causal processes by teasing out their multiple, fluid and relational elements. The article goes on to consider new ways to broker the interface between research endeavours and policy processes, based on collaborative modes of knowledge co-production that unfold in real-time. QL enquiry emerges here as more than an added option, a 'niche' approach. It has potential as a valuable 'impact' tool for discerning causality in new ways and reformulating insights into 'what works?', with scope to play a pivotal role in shaping and facilitating change in policy and practice settings.

Keywords: Qualitative longitudinal research, fluid enquiry, complex causality, co-producing knowledge, QL impact research.

There do not exist things made, but only in the making, not states that remain fixed, but only states in process of change ... [We] arrive at fluid concepts, capable of following reality in all its windings. (Bergson, 1946 [1903]: 188, 190)

[Class] is not a thing, it is a happening ... not this or that part of the machine, but the way the machine works once it is set in motion ... the friction of interests - the movement itself, the heat, the thundering noise. ... the definition can only be made in the medium of time, that is, action and reaction, change and conflict.' (E. P. Thompson, 1978: 85)

Our sense of time involves our ability to separate cause and effect, means and end.... And there morality enters in [and] responsibility. ... [W] are creatures of time ... a complexity that includes not only duration but creation, not only being but becoming, not only geometry, but ethics. It is not the answer we are after, but only how to ask the question. (Le Guin, 2019 [1974]: 187)

\section{Introduction}

In recent years, researchers have begun to address questions about the value of Qualitative Longitudinal $(\mathrm{QL})$ research and what it may reveal about real-world processes. Taking up these themes, and drawing on a more detailed discussion (Neale, 2021), this article explores two aspects of QL enquiry: firstly, its power to illuminate complex causal processes and, secondly, its capacity to support and facilitate the production of dynamic, real-world evidence for policy and professional practice. The article begins with a brief introduction to QL research, outlining its distinctive features as a form of fluid, processual 
enquiry, its foundations in the interpretivist tradition of research, and its power to trace and respond to the flux of lives as they unfold. The longitudinal frame and flexible, responsive tempo of QL research creates a dynamic landscape for 'walking alongside' research participants, and 'following reality in all its windings' (Bergson, 1946 [1903]; Neale, 2021). It enables understandings not only of what changes, when or by how much (the measurement of change), but how change is created, negotiated, lived and experienced (the situated meaning and significance of change). As our opening quotations show, these more nuanced understandings of causal processes have been recognised and debated by key thinkers over many decades.

The article goes on to explore the complexities of causality, drawing on the distinction between 'simple' and 'complex' forms of causal logic (Byrne et al., 2009). It moves beyond linear, variable-based, snap-shot understandings, to highlight the multiple, fluid and relational dimensions of causal processes. Drawing together insights from $\mathrm{QL}$, realist and social complexity research, the discussion highlights the value of discerning causality through a fluid, processual, interpretive and relational lens. The grounded, real-time nature of QL methodology offers new ways to think about the causes and consequences of change in real-world settings, and of reframing the policy mantra of what works? by considering how things work? in different contexts of time and space (Neale, 2021).

The final part of the article considers how QL methodology may be harnessed to support and facilitate real-world policy processes. It explores the potential use of QL impact research as a design and navigational tool in the development of policy interventions. The discussion suggests new ways to broker the complex interface between research endeavours and the multiple concerns and drivers of policy and professional practice. The overall aim is to produce new insights into the nature of causal processes from a QL perspective, and to illustrate the capacity of this methodology to play a pivotal role in rethinking 'what works?' for policy and professional practice.

\section{$Q L$ research: fluid enquiry and the processual turn}

QL research typically takes the form of small-scale, in-depth studies of individuals or collectives, generating rich, situated data about unfolding lives. With its dual identity (longitudinal and qualitative) it spans two established methodological traditions. Firstly, as a small but vital component of the longitudinal canon, it seeks to follow the same individuals or groups (e.g. a panel of welfare recipients, service providers and/or policy makers) prospectively, in real-time, to capture 'change in the making' (Wright Mills, 1959). QL research also incorporates a valuable retrospective lens. This enables participants and researchers to trace processes forward and backward in time. The process is iterative, involving the application of abductive logic (detailed in Neale, 2021). We are afforded insights not simply into what happens over time, but how processes unfold, and the meaning they hold for those experiencing them. How questions are inherently processual. Moving beyond simple, clear-cut what questions, they are crucial in understanding why things unfold in particular ways.

Secondly, located within the interpretivist tradition of social research, and as part of the broad and porous canon of socio-historical studies (longitudinal ethnography, restudies, biographical, life history and narrative research), QL research explores dynamic processes through an in-depth, qualitative lens. This is real-world research, which gives insights into how people narrate, understand, practice and shape their evolving lives, and 
the evolving world of which they are a part. QL research takes human agency and subjectivity seriously, enabling understandings of lived experiences, and gaining an intimate familiarity with lives as they are being lived.

The qualitative and longitudinal elements of QL methodology are entwined and captured in the notion of 'walking alongside' people. By turning a snap-shot of social life into an intimate 'movie', it becomes possible to uncover how lives are lived and experienced, as well as how they are narrated (Neale, 2021). The flexibility of this methodology, and its responsiveness to change, gives QL research a unique capacity to mirror the flux of the world, to follow processes as they unfold (Neale, 2021). This flexibility is vital to its methodological rigour, ensuring that a QL study is in tune with and able to reflect dynamic, real-world processes. It is through a deep and sustained engagement in the field that complex processes can be brought more readily to light (Langley et al., 2013).

QL enquiry has developed over recent decades as part of a widespread processual 'turn' in social enquiry. This has brought a shift in focus from the form that social events take, their structural components, to their relational and dynamic elements. As Bergson and E. P. Thompson saw, it is the dynamic relationship between things that matters, not the things themselves. This shift in thinking from structures to processes has seeped into the very foundations of interpretive enquiry, and is set to provide an important bridge between positivist, realist and interpretivist research traditions (Neale, 2021). As part of this processual turn, QL enquiry has gradually consolidated its credentials as an established and creative methodology that straddles the longitudinal (real-time) and qualitative (real-world) canons. This is reflected in a rapidly expanding body of methodological literature, and a growing sophistication in how this approach is being conceptualised and applied (reviewed in Neale, 2021).

While QL social policy research has a long history, in recent years there has been a rapid uptake in policy-related research across a range of fields: for example, in health, housing, education, social work, criminology and environmental issues (Corden and Millar, 2007; Neale, 2021). This growth is hardly surprising. An understanding of change, continuity and causality is vital in policy domains where people are required or encouraged to change their practices, to adapt to changing circumstances, or to develop their knowledge or learn new skills. It is no less vital where the effects of policy interventions need to be monitored and evaluated (Corden and Millar, 2007).

\section{Fluid time}

The powerful features of QL enquiry outlined above reflect the underlying theoretical drivers for this mode of research: it is rooted in fluid, interpretive notions of time that go beyond the fixed regularities of the clock and calendar (Bergson, 1910 [1889]; Adam, 1990). From an interpretive perspective, objective, constant, linear, one-dimensional time gives way to a plurality of times, held in a simultaneous relationship with each other, which flow and intersect in multiple ways. Fixed time is perceived as an overarching external force, a constant unvarying presence within which events take place and lives unfold (an 'events in time' perspective). Fluid time, in contrast, is experiential and socially constructed. It is embedded in who we are, inhering in our everyday sensibilities, events, practices and processes (a 'time in events' perspective, Bergson, 1910 [1889]; Adam, 1990). While fluid time does not replace fixed, linear time, in this broader formulation, 
linear clock-based time loses its dominance. It becomes one among the many flows of time that constitute our temporal world, and that shape our lived experiences (Adam, 1990; Neale, 2015, 2021).

How events and processes are perceived in social enquiry depends on how time itself is perceived. For example, viewing change through the lens of fluid enquiry has a transformative effect. The ontological focus shifts from change as something that happens to things, to change as an inherent part of the nature of things. Social phenomena of all kinds take on a new meaning when viewed through the prism of fluid time. From a fixed, linear perspective, for example, a process is an orderly set of events that unfolds sequentially, with a clear purpose, discrete markers along the way, and a definitive end point (see Charmaz, 2014: 17 for this orthodox view). From a fluid perspective, however, processes can be understood as rich streams of linked events, contexts, actions, reactions and interactions that are intertwined, and that unfold in non-linear and unpredictable ways (Neale, 2021). As will be shown below, similar transformations occur in our understandings of causality when viewed through a fluid lens.

In sum, studying lives qualitatively through time is not a straightforward matter of creating a moving, chronological picture that charts observable changes and discerns what happens next. Lives do not necessarily unfold in chronological order, through discrete changes, in one linear direction, or at a uniform pace. Nor do people experience time in these fixed, linear ways (Neale, 2021). QL research alerts us to an ever-changing kaleidoscope of lives in the making. In the process, the messy, complex, volatile, contradictory, shifting facets of human experience are brought to light (Farrall et al., 2014). It becomes possible to explore the stability or inertia of continued states; the triggers, driving forces and tipping points for change; and the creativity, resilience and/or fragility of individuals and groups in shaping or accommodating to these processes. It is this capacity for fluid enquiry, underpinned by a fluid understanding of time, which gives QL research its rich explanatory power (Neale, 2021).

\section{Causal processes}

Having set out the broad contours of QL research, we now turn to the nature of causality. The focus here is on how causal processes may be understood from a fluid, qualitative perspective. The search for causal explanations, an understanding of cause and effect, is deeply rooted in social enquiry, and in the flux of everyday life (Maxwell, 2012). Causality is our way of making sense of the world, and, as Le Guin notes (above), of giving shape to our moral choices and responsibilities. In the Aristotelian tradition, 'causes' are simply explanations, forms of reasoning that give meaning to our existence. In general, we want to know how things happen and why events and actions turn out in a particular way. Indeed, our credentials as researchers often depend upon our ability to explain these deeply embedded processes, although we may not use the language of causality (Lofland et al., 2006: 159; Hammersley, 2008). Research findings are likely to contain implicit causal reasoning, for 'causal statements are pretty much inevitable in any discussion of human social and cultural life' (Seale, 2018: 573). As Maxwell notes, 'any argument that something 'influences' 'impacts', 'shapes' or 'transforms' something else is a causal claim' (Maxwell, 2012: 42). Moreover, 'unless qualitative researchers are able to talk in cause/ effect terms they will not have much to say to a policy community focused on the question, 'what works?' (Anderson and Scott, 2012: 680; Donmoyer, 2012). 
Despite this, causality has long been a contested issue in the interpretive tradition of research. Positivists have traditionally held the view that qualitative researchers should stick to descriptions of the world, and leave causal explanations to those working with large datasets that lend themselves to measurement based, statistical modelling and large scale generalisations (Maxwell, 2004). Until recently, this paradigmatic 'stand off' was also evident among qualitative researchers. The search for cause and effect was seen as a defining feature of predictive, measurement-oriented positivism, and not, therefore, a valid enterprise for interpretive enquiry: 'the concept of causality is so beleaguered and in such serious disarray that it strains credulity to continue to entertain it in any form approximating to its present (poorly defined) one' (Lincoln and Guba, 1985: 141). Qualitative understandings of causal processes were set back for decades, through fears that the findings would be tainted by their positivist associations (Maxwell, 2004; Hammersley, 2008).

\section{Simple causality}

Part of the problem is that the language of causality and of causal mechanisms conjures images of conveyor belt precision and narrow, instrumental, mechanistic processes that may bear little relationship to the flux of the real world. The variance/inference causal logic used by positivists produces a simple understanding of causality that relies on a combination of two temporal flows. The first is chronology ( $\mathrm{A}$ is followed by $\mathrm{B}$ ). The second is causality (A causes $B$ ). The logic holds that if $A$ is typically followed by $B$, then $A$ must cause $B$, particularly where this pattern occurs regularly and repeatedly across large samples (Abbott, 2001; for a more detailed account see Lofland et al., 2006). Since causality cannot be directly observed, it can be inferred from two or more snap-shot visions of events, a string of discrete moments in time (temporal variables), which are held together (correlated) by their consistently observed sequence in a linear chain. Causality is implied in the linear, orderly progression from past to present to future; cause and effect are intimately tied to this sense of chronology. Viewed as a simple, predictive, instrumental relationship between cause and effect, causality is reduced here to regularity, repetition and sequence (Maxwell, 2012: 37).

Some blistering attacks have been levelled at this inferential logic. Sayer (2000: 13), for example, astutely observes that what causes something to happen may have very little to do with the number of times we observe it happening. The link between causality and repetition and regularity is far too superficial and tenuous. Moreover, on its own, the bald fact that $A$ is followed by B may also tell us very little. In much formal sequential analysis there is too little room to explore the discontinuities, irregularities, sudden accelerations, trigger points and pivotal moments of change, or to aim for a more rounded and nuanced understanding of how $A$ is linked to $B$. The limitations of simple, linear understandings of causality, which create a series of snapshots of change, have been extensively critiqued. QL organisational researchers, for example, point out the 'difficulties we face when we try to understand change by breaking it down to stages. By doing so, change is reduced to a series of static positions - its distinguishing features are lost from view' (Tsoukas and Chia, 2002: 571; Gerrits, 2008; Langley et al., 2013; Langley and Tsoukas, 2017).

Similarly, Abbott (2001: 192) observes that events or occurrences have duration and may overlap, raising questions about the conceptual order or sequencing of things. This is 
related to the 'time horizon' problem (Abbott, 2001: 193): how do we account for processes that involve multiple, contingent sequences of events that are moving at different speeds, across different scales of the social fabric? In short, how far is it possible to tease out the multiple interlocking threads of a process, occurring at varied and overlapping tempos? While we can discern a great deal from the intricate patterning of events and interactions, with their different temporal rhythms, the sequence itself is likely to form just one element in a much more fluid picture.

Of course, this is not to deny the critical importance of studies that discern widespread correlations between discrete social phenomena. For example, the simple link between smoking and ill health, established in the 1950s, has led to a raft of in-depth investigations into how such correlations actually arise. Taken together, these studies reveal a host of factors (diet, life style choices and broader socio-economic and environmental inequalities) that impact on health, suggesting that a complex intertwining of individual, social and structural factors is at play. Mixed longitudinal studies, which combine numerical and narrative forms of explanation, can provide depth of understanding alongside a broader vision of socio-historical processes (Neale, 2021).

Nevertheless, where variance/inferential causal logic is used as the sole source of evidence, there is a danger of producing flawed causal models that are too superficial, tenuous, narrow or spurious to be of value (Maxwell, 2004; Anderson and Scott, 2012; Westhorp, 2018). The drawback is that simple causal models are spatially and temporally adrift, 'discovered from no-where' (Hartsock in Frankham, 2009: 2). They are too abstracted from real-world processes, practices and interactions, and the meanings and motives of those involved.

\section{Complex causality}

In recent decades, a more fluid, processual understanding of causality has emerged as an alternative to the variance/inference approach outlined above (Maxwell, 2004, 2012). This new thinking has emerged as part of the processual turn in social enquiry. It recognises the 'openness, contingency and contextually variable character of social change' (Sayer, 2000), and seeks to discern how causal processes operate and unfold in real-world settings (Maxwell, 2004; Donmoyer, 2012). In policy terms, a processual approach seeks to understand not simply what works? but what works for whom, under what circumstances and why' (Pawson and Tilley, 1997; Pawson, 2006). This more recent thinking has wrested causality from its positivist stronghold and made it a legitimate concern for qualitative enquiry (Maxwell, 2012).

A processual approach to causal understanding has developed across numerous studies. It is a cornerstone of QL enquiry (Pettigrew, 1995, 1997; Gerrits, 2008; Langley et al., 2013; Bidart et al., 2013; Langley and Tsoukas, 2017); and of realist research (Pawson and Tilley, 1997; Sayer, 2000; Pawson, 2006; Maxwell, 2004, 2012; Byrne et al., 2009; Emmel et al., 2018). It has also formed the foundation for complexity theory, a powerful amalgam of ideas drawn from systems theory, social network analysis and chaos theory (Cilliers, 1998). Varied ways of understanding and investigating causal processes have developed within and across these different research traditions. While there is no one settled or integrated methodological approach, several important synergies and differences are worth teasing out here. 
Firstly, QL researchers tend to produce detailed longitudinal case studies to show how causal processes unfold (Pettigrew, 1995; Gerrits, 2008; Bidart et al., 2013). Realists, in contrast, tend to develop theoretical models in order to illuminate and predict the regularities and patterns of causal processes, and the underlying social mechanisms that drive them (Byrne and Callaghan, 2014). A standard formula is commonly applied, which seeks to model contexts, mechanisms and outcomes (Pawson and Tilley, 1997; Pawson, 2006), although this formula is acknowledged to be overly rigid and ripe for modification (Emmel et al., 2018). There is also the view that such predictive modelling runs the risk of becoming reductionist and hypothetical, of ironing out the complexities of processes in ways that are too abstract and simplistic (Hedström and Swedberg, 1998; Hedström and Ylikoski, 2010).

Secondly, alongside these investigative and analytical differences, there are variations in how processual researchers engage (or fail to engage) with time. Much like QL researchers, social complexity theorists recognise and work with fluid understandings of time and causality (Cilliers, 1998; Gerrits, 2008; Boulton et al., 2015; Neale, 2021). For Boulton et al. (2015), seeing the world in terms of complex fluidity is closer to lived experience than the Newtonian idea of the world as a machine. They suggest ways to research the complex world, in all its grittiness and granularity, 'not through mathematical models and theories but 'in the flesh' through 'being in it' (Boulton et al., 2015: 107). They advocate process tracing as a means to identify chronologies and 'the causal links and pathways which lead actions through to impact' (Boulton et al., 2015: 190; Neale, 2021). They also recommend working across fixed and fluid, sequential and non-sequential time, and working both prospectively and retrospectively to piece together a processual picture. Moreover, they promote qualitative, narrative, case history, action research and longitudinal methodologies as the prime means to achieve these goals. Their general advice is to be processual: to follow processes and anticipate tipping points, to trace and represent the development of situations over time; to allow for detours along the way, and to be open to multiple perspectives through a critical subjectivity (Boulton et al., 2015: 112-14).

Realist researchers, in contrast, rarely conduct their studies through time, or engage with the fluidity of time. Models of causality are usually developed with no more than a passing reference to time, or, rather, with an implicit understanding of time as a fixed, linear entity (Westhorp, 2018: 45). Yet when fluid time is brought into the picture, the nature of realist modelling is transformed. The notion that there are discrete mechanisms, contexts and outcomes that can be separated out sequentially for analysis becomes suspect. The temporal location of these causal threads is continually shifting, such that an effect (or outcome) operating one moment in time, may become a cause, a contextual factor or a mechanism at another (Westhorp, 2018). Influences, then, may run in more than one direction, giving cause and effect a circular rather than chronological relationship; the formula A leads to B is transformed where A leads to B leads to A (Byrne and Callaghan, 2014). The very idea of discrete causes, contexts, mechanisms or outcomes becomes problematic in this shifting, fluid landscape.

Such differences, however, do not detract from the important commonalities that exist across these fields of enquiry, and the potential to draw on a rich amalgam of ideas to produce new processual insights. Taking up this opportunity, an attempt is made here to tease out and untangle the multiple, fluid and relational elements of complex causality. 


\section{The multiplicity of causal processes (thick causality)}

Perhaps the most evident feature of complex causality is that it is driven by an intricate web of interacting elements that make up the social world. Multiple causal threads unfold in any one process, spreading across varied spatial and temporal settings, and operating at different scales of the social fabric (local to global, individual to social, micro to macro) (Pettigrew, 1997; Tsoukas and Chia, 2002; Langley and Tsoukas, 2017; Pawson and Tilley, 1997; Sayer, 2000; Maxwell, 2012). These multiple threads are perpetually flowing, oscillating and interacting through time; they are intertwined like a confluence of streams flowing through a river basin or across a flood plain (Pettigrew, 1997). They are shaped by driving forces and feedback or feedforward loops that may converge at key moments in time: to reaffirm, grow, adapt or otherwise transform the social fabric (Cilliers, 1998; Gerrits, 2008).

The influences that produce change, or lead to recurring states are not only multiple and flowing in all directions. They are also distributed (Cilliers, 1998). In complex systems there will be many pockets of differential power and influence, but there are no fixed levels, control points, stable contexts, and no enduring external designers that can, with any certainty, determine the nature, direction and impact of change, or shape exactly how or when it will occur. This helps to explain why policy directives may fail or have unintended consequences. Given their distributed nature, complex processes are unpredictable: actors working in one part of the system will not be fully aware of the range or impact of their actions and interactions in another (Cilliers, 1998; Gerrits, 2008). And neither will researchers. Causality is recast here. In the maelstrom of complex social processes, there is no simple or unitary relationship between a cause and effect. Indeed, there are no discrete causes that lead to discrete effects, in clear cut directions, over discrete sequences of time. Tracing effects back to a single cause is far too simplistic when they are rooted in entangled webs of causality (Nowotny, 2005).

The aim of QL research is to capture this dynamic reality in flight (Pettigrew, 1997): to follow processes where they lead, identify their constituent threads, and discern how these threads are connected and how they coalesce to create the momentum for change. This is 'thick' causality (Fleetwood, 2014), which 'does not follow some homogeneous if-when formula ... but takes a multiplicity of different forms' (Porpora, cited in Dall and Danneris, 2019: 589). In policy terms, it becomes clear that 'what works' is shaped by the particular circumstances and contexts in which change occurs. A failure to generate holistic, empirically-driven and locally situated understandings risks producing policy recommendations that present a narrow, partial or abstract picture, or that treat the symptoms rather than addressing root causes (Anderson and Scott, 2012: 679). These varied insights suggest the need for pluralism and empirically grounded sensitivity in how causal investigations and explanations are framed (Maxwell, 2004).

\section{The fluidity of causal processes}

Processes convey a sense of flux and change: of becoming, development, emergence, progression, evolution, revolution, transformation, dissipation, decay and so on (Lofland et al., 2006: 152). If causal processes are multiple, operating through different settings and scales of the social fabric, they are also inherently dynamic. Moreover, the relationship between cause and effect is not a simple matter of chronology: processes emerge and are 
shaped through the fluidity of time. If tracing back to a single cause is too simplistic, so too is tracing back through a clear-cut, linear sequence of events: 'Causality is neither singular nor linear. ... Changes have multiple causes, and are to be explained more by loops than lines' (Pettigrew, 1995: 96).

The temporal dimensions of processes increases their multiplicity. Every event or experience has multiple antecedents, spiralling backwards through time, and multiple consequences, spiralling forwards into the future, creating vast and ever expanding constellations of causal flows (Abbott, 2001: 144, 192). Linearity here gives way to multi-directional zigzags, recursive loops, and processual patterns that may be cyclical, occur at random, spiral up and down, or otherwise grow and dissipate through time. This means that the course of a pathway, its trajectory, patterning and momentum, plays a part in shaping how events unfold. In other words, the nature of the journey assumes just as much importance as the starting point, or the destination reached (Neale, 2021).

This fluidity brings to the fore the inherently contingent, unfinished and unpredictable nature of causal understandings. Causal processes are not located in static environments or contexts; there are no stable or external 'wrappers' within which change unfolds. Processes have no temporal fixity, no discrete start and end points, other than artificial ones that are created as an artefact of the research process and its window of observation. As Abbott (2001: 145) notes, when we think through time, the idea of beginnings, middles and ends quickly gives way to a sense of endless middles.

These insights into how causal processes unfold have significance for policy and professional practice. Rather than a focus on 'what works?', we need a fluid, processual understanding of how things work through the stream of time. As Stich and Cippolone (2017) observe in their QL study of urban school reforms in the US, 'it is not just the passage of time that is important ... but also the accumulations, recursions, connections, relations, stabilizations and destabilizations that develop ... as time passes' (Stich and Cippolone, 2017: 5). Discerning this fluidity in unfolding processes is greatly enhanced through indepth, real-time, sustained field investigations, of the sort afforded by QL research.

\section{The relationality of causal processes}

This is the third key feature of complex causality. Complex causal processes are not only multiple and fluid. They are inherently relational and infused with ethical sensibilities (Le Guin, 2019 [1974]). There is a growing recognition that causal forces do not reside in social structures per se, but in the complex interaction between individuals and the collectives of which they are a part (Harrē, in Blaikie and Priest, 2017). As Sayer observes, '... explanation requires mainly interpretive and qualitative research to discover actors' reasoning and circumstances in specific contexts - not in abstraction from them' (Sayer, 2000: 23). Human agency (the capacity to act, to interact, to influence the shape of one's life and the lives of others), and subjectivity (the shifting meanings that events and processes hold for those who experience and craft them) are central to causal understandings (Hammersley, 2008; Maxwell, 2012; Byrne and Callaghan, 2014; Neale, 2021). As Hammersley (2008) notes, the very notion of agency is founded on an understanding of the causal powers of individuals and groups.

These insights suggest the need for further reformulations of the 'what works?' mantra. Understanding how things work requires a relational understanding of what works for whom, bolstered by a prior understanding of what matters to people, and what helps them 
in particular circumstances. Gaining insights into lived experiences is vital in understanding causality, and in generating 'know how' knowledge (Langley et al., 2013: 4). These more nuanced, situated, consultative, 'insider' forms of causal knowledge avoid a narrowly prescriptive and instrumental focus on 'what works?'. As a broader, overarching concept, complex causality encompasses simple causality, rather than replacing it. In fleshing out the picture, it provides essential insights into the causes and consequences of change.

The discussion above suggests the value of finely-grained longitudinal case data in generating insights into causal insights. To give just one example, the relational and fluid elements of complex causality have been explored recently in a two-year QL study of a Danish welfare-to-work programme and its impact on vulnerable clients (Danneris, 2018; Danneris and Caswell, 2019). The researchers followed the clients' journeys through the programme, documenting how it was delivered 'on the ground', and discerning its effects, including the mental health effects of a programme driven by a rigid and punitive welfare ideology. The researchers developed a typology of client trajectories: progressing, deteriorating, stagnating and derailing (which, in some cases, included suicide or attempted suicide). These different journeys were fluid and unpredictable (rather than linear or straightforward), marked by fits and starts, zigzag paths between different trajectories, and recurring spirals of hope and despair (Danneris and Caswell, 2019). It was not possible to predict when a client might enter into a downward spiral, or reach a tipping point into derailment. But these patterns were most often linked to the punitive sanctions of the programme, or the 'quick fix', 'one-size-fits-all' nature of the work placements, which were commonly tenuous, unsuitable, unsustainable and demoralising (Dall and Danneris, 2019).

Retrospective evidence on what led to sustained employment for these clients revealed that programme strategies (structured activities, a rule-bound system and sanctions to enforce compliance) were less effective than the positive relational elements that some practitioners managed to build into the programme: sustained and flexible support, and tailored, responsive provision based on the values of respect, encouragement, and a sense of partnership in a collaborative project (Danneris and Caswell, 2019). The authors also tease out the intertwining of individual and structural factors in these unfolding processes, showing how clients and service providers may be 'pushed down particular streams' by external forces beyond their control (Davidson, pers. comm.) Similar findings on relational causality, alongside fluid narratives of contingency, chaos and/or liminality, have been reported in a range of QL studies that explore the lived experiences of service users (Dearden et al., 2010; Ridge and Millar, 2011; Pinnock et al., 2011; Neale and Davies, 2015; Patrick, 2017; Ferguson et al., 2020; Dwyer, 2018).

\section{Methodological challenges}

Exploring causality 'on the ground' in the ways suggested above is not without its challenges. There is the complex task of uncovering the multiple threads that lead to causal effects, and discerning their relative salience in shaping lives. Since causal processes are contingent, unpredictable, and inherently unfinished, we must capture them 'in flight', through small windows in time, and in situated corners of the social fabric. The aim is to produce plausible interpretations that are acknowledged to be inherently provisional and in the making. This requires greater modesty in our claims to causal 
understanding, a need to tie causal explanations of actions and reactions, effects and counter-effects much more closely to grounded, subjective and retrospective accounts (Cilliers, 1998).

A related challenge concerns the common misconception that only statistically based, or more theoretically driven studies have the capacity to generalise and 'scale up' their findings (Anderson and Scott, 2012; Donmoyer, 2012; Dall and Danneris, 2019). In fact, qualitative forms of generalisation are well established (Gobo, 2004; Neale, 2021), although they could be more effectively utilised, packaged and promoted. Unlike systematic reviews of strictly comparable statistical data, qualitative generalisations allow for a mosaic of complementary evidence to be woven into a composite picture, including insights drawn from legacy datasets (Neale, 2021). With each new addition to the analytical 'pot', the conceptual picture is enhanced or transformed. In this mosaic approach to evidence building, insights about patterns and processes of change are given added credence through the extent, variety and weight of the evidence base, and the strategic manner in which comparisons and connections are drawn out (Halford and Savage, 2017; Anderson and Scott, 2012; Donmoyer, 2012; Monaghan and Boaz, 2018; Dall and Danneris, 2019; Wright and Patrick, 2019; Middlemiss et al., 2019). For busy policy makers, such evidence has the potential to create conceptual impacts, to raise new questions and to change perceptions of how things work, as a necessary precursor to creating instrumental changes in policy and professional practice (Neale and Morton, 2012; Monaghan and Boaz, 2018).

\section{Evidence-based policy?}

A further and equally pressing challenge also arises: how to make dynamic evidence about complex causal processes count in policy decision making? Currently, the research evidence base is used highly selectively to address 'what works?' questions. In part, the issue arises within the academy. The preference is for 'hard' economic or statistical evidence, such as randomised controlled trials, meta-evaluation, quasi-experiments and quantitative statistical testing, along with systematic reviews of such research (Dall and Danneris, 2019; Monaghan and Ingold, 2019). Such evidence is placed at the top of an evidence 'hierarchy' on the grounds that it is scientifically robust, while 'soft' qualitative and case study evidence, which forms the bulk of the research canon, is relegated to the bottom, where it can be effectively side-lined. This hierarchy is currently entrenched: 'to challenge variable-centred approaches ... is to challenge a whole power structure in the academy' (Byrne et al., 2009: 519-20).

A preference for 'hard' data is also found within organisational cultures and capabilities, which are adapted to working with simple, variance-based causal models (Monaghan and Ingold, 2019). With their simple inputs and outputs, these data can be translated with relative ease into policy directives. Working with complexity is often anathema to the clear-cut needs of policy makers. This is the case despite the fact that evidence rooted in simple causal models is consistently reported to be ambiguous and inconclusive in meeting real-world policy objectives, or in identifying what actually works (Pawson, 2006; Byrne et al., 2009; Donmoyer, 2012; Millar and Bennett, 2017; Dall and Danneris, 2019; Monaghan and Ingold, 2019). As the discussion above shows, the ambiguity of such evidence is hardly surprising if it is based on the tenets of simple causality i.e. on assumptions that a system input will, in instrumental, mechanistic or deterministic ways, 
lead to a desired outcome; and that the presence of an input will be the necessary and sufficient condition to bring about change (Dall and Danneris, 2019).

Of equal concern is an evident dumbing down of expert research evidence as a valuable base upon which to shape policy. The whole edifice of UK evidence-based policy, which developed in the late 1990s under New Labour, appears to be crumbling, as ideological drivers (the austerity agenda, the conditionality of state aid and support, and centralised decision making and control) take precedence: 'Rationality and logic are often trumped by other considerations ... bigger forces can easily relegate research to a bit player (or no player at all)' (Sainsbury, 2012: 373). The result is a growing chasm between empirical evidence and policy-making (Millar and Bennett, 2017; Patrick, 2017; Dwyer and Patrick, 2020). What counts as evidence, then, is based on what is deemed permissible and helpful to policy. While, ostensibly, the evidence shapes policy, policy is just as likely to shape the evidence to its own ends (Millar and Bennett, 2017; Monaghan and Ingold, 2019).

\section{Re-configuring the interface between research and policy}

Whether QL research can contribute to understandings of 'what works?' in such a climate is an open question. It would require some subtle shifts in the way policy processes are configured, along with greater clarity and consensus about what constitutes robust evidence for policy, and how it may be obtained and translated (Dall and Danneris, 2019; Monaghan and Ingold, 2019). Whether complex causality is recognised and valued may depend, too, upon its conceptual impact; on how this alternative way of seeing things can be packaged and presented to policy makers as a formula that can be operationalised. This, in turn, depends on new thinking about ways to broker the interface between research evidence and complex policy processes (Kislov et al., 2017).

In recent years, researchers have made some strides in addressing this issue. The late twentieth century principles of knowledge transfer (a one-way, linear approach) and knowledge exchange (two-way, but equally linear) have gradually been incorporated into a more systemic, iterative and integrated model of knowledge co-production (Best and Holmes, 2010). This fosters policy-informed research and research-based practice (Neale and Morton, 2012). It is an approach predicated on collaborative modes of policy formulation and implementation that are less 'top down' and ideologically driven, and that allow for active partnerships between researchers, policy makers, and service providers, along with the participation of service users (Frankham, 2009).

The credentials of such an approach have been established through action research and participatory methodologies, which do not simply research change, but which seek to facilitate change (Bradbury Huang, 2015). Action research is inherently dynamic, flexible and cumulative, a process involving iteration between the research process and practical action (Figure 1). In a parallel development, partnership and design-based models of research are beginning to appear, for example, in the fields of health and education (Penuel and Gallagher, 2017; McKenney and Reeves, 2018; Nyström et al., 2018). These accord a more collaborative and integrated role for research in designing, co-ordinating and implementing policy programmes, as well as evaluating them. 


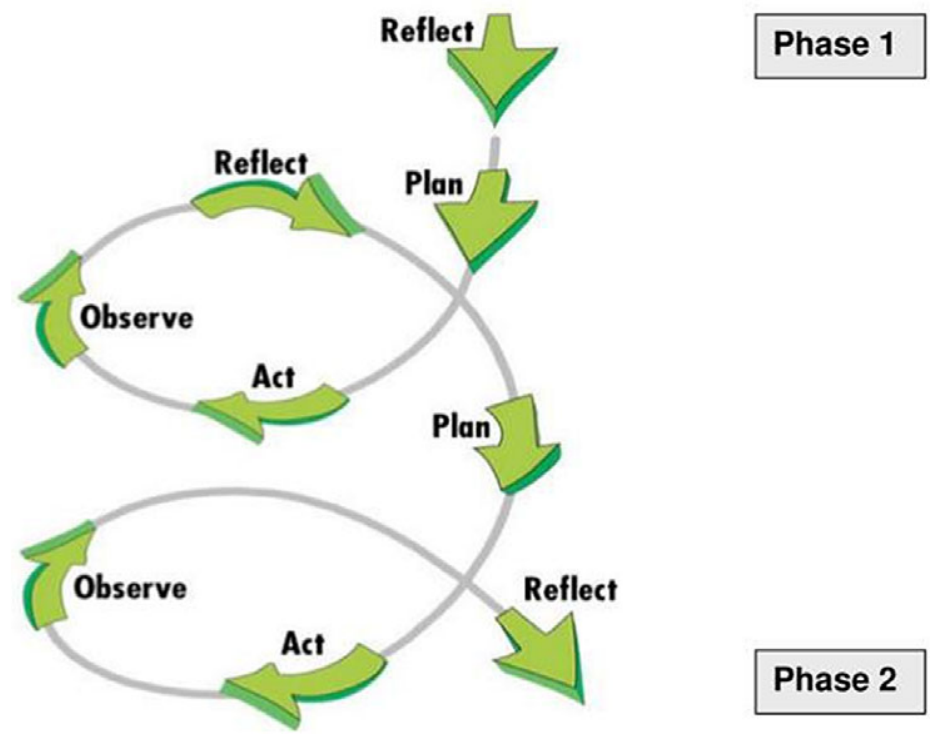

Figure 1. The Action Research Spiral (Kemmis and McTaggart, 1988).

\section{QL impact studies}

As part of these developments, QL impact research offers innovative ways to broker the interface between research and policy/practice developments, based on collaborative real-time modes of knowledge production. With its flexible, grounded, responsive and interactive tempo, QL research can act as a design, co-ordinating and navigational device, which helps to shape policy interventions and then 'walks alongside' to uncover how these processes are unfolding. Indeed, the real-time iteration between research and policy processes enables stakeholders to take stock at key moments, try out new directions, and refine the elements of a programme as it unfolds, in ways that may create and stimulate change. An exploration of how things work is embedded within and emerges through the research process.

Drawing on design-based methodologies (McKenney and Reeves, 2018) and their application in QL research (Kinsey, 2020), a QL impact design might include the following:

1. Stakeholder Consultations to identify gaps in knowledge, perceptions, current limitations, possible solutions among policy makers, analysts, and front line services; recruitment of policy/practice partners and client consultants;

2. Integrative review and synthesis of complementary research evidence/ legacy data, developed in consultation with stakeholders;

3. Collaborative Design of a new intervention; production of practice protocols;

4. Pilot Programme implementation: using intensive longitudinal methods to track, monitor, review and evaluate the new initiative;

5. Case Study reporting/review of potential modifications and further developments in different contexts/settings. 
6. In a two-phase or rolling research design, an initial tracking and evaluation of existing provision can become a spring board for the design, development and evaluation of a new initiative. Indeed, in line with the action research spiral (above). the process allows for successive waves of reflecting, planning, acting and observing, in a cumulative process of review and refinement.

The organisational and relational challenges of $\mathrm{QL}$ designs based on partnership working should not be underestimated. Creating a porous and collaborative interface between research and policy/practice requires brokering skills to secure and sustain 'buy in' from busy professionals (Monaghan and Boaz, 2018). It also requires clear terms of reference, diplomacy and agility to maintain a balance between the competing claims and perspectives of multiple stakeholders (Flinders et al., 2016; Penuel and Gallagher, 2017; Kislov et al., 2017; Nyström et al., 2018; Kislov, 2019). Nevertheless, these forms of research are being effectively used by QL researchers, and they offer considerable power and potential to make a difference in policy and practice settings (Kislov, 2019; Kinsey, 2020, showcased in Neale, 2021).

\section{Conclusion}

The elements of complex causality outlined in this article reinforce a basic insight: causality, as a complex, plural, fluid and relational force, is not something that happens mechanically as processes unfold; it is embedded in the way processes unfold. We have seen that it is possible to mirror and trace complex causal processes using the real-world, real-time methodology of QL research. Researching the multiple, fluid and relational dimensions of causal processes enables fresh insights into how things work in particular contexts and settings of change. Moreover, using QL research as a design, co-ordinating and navigational tool means that causal processes are not simply documented or observed, but are actively shaped as the research unfolds. Either way, the research is more effectively embedded within policy developments, while impact is not 'tacked on' to the end but becomes an integral part of the research process. These capabilities suggest that QL methodology is more than an added option, a 'niche' approach. It is a valuable tool for understanding a dynamic world, crafting ways forward, reconfiguring 'what works?' and generating useful 'know how' knowledge for policy and professional practice.

\section{Acknowledgement}

The author is grateful to two anonymous referees for their helpful comments on an earlier draft of this article.

\section{References}

Abbott, A. (2001) Time Matters: On Theory and Method, Chicago: University of Chicago Press. Adam, B. (1990) Time and Social Theory, Cambridge: Polity.

Anderson, G. and Scott, J. (2012) 'Towards an intersectional understanding of process causality and social context', Qualitative Inquiry, 18, 8, 674-85.

Bergson, H. (1910 [1889]) Time and Free Will (trans. F. Pogson), London: George Allen and Unwin. Bergson, H. (1946 [1903]) 'Introduction to metaphysics', in H. Bergson, The Creative Mind, New York: Citadel Press, 159-200. 
Best, A. and Holmes, A. (2010) 'Systems thinking, knowledge and action: towards better models and methods', Evidence and Policy, 6, 2, 145-59.

Bidart, C., Longo, M. and Mendez, A. (2013) 'Time and process: an operational framework for processual analysis', European Sociological Review, 29, 4, 743-51.

Blaikie, N. and Priest, J. (2017) Social Research: Paradigms in Action, Cambridge: Polity.

Boulton, J., Allen, P. and Bowman, C. (2015) Embracing Complexity: Strategic Perspectives for an Age of Turbulence, Oxford: OUP.

Bradbury-Huang, H. (2015) The Sage Handbook of Action Research, London: Sage.

Byrne, D. and Callaghan, G. (2014) Complexity Theory and the Social Sciences, London: Routledge.

Byrne, D., Olsen, W. and Duggan, S. (2009) 'Causality and interpretation in qualitative policy-related research', in D. Byrne and C. Ragin (eds.), The Sage Handbook of Case-Based Method, London: Sage, $511-21$.

Charmaz, K. (2014) Constructing Grounded Theory 2nd edn, London: Sage.

Cilliers, P. (1998) Complexity and Postmodernism: Understanding Complex Systems, London: Routledge.

Corden, A. and Millar, J. (eds.) (2007) 'Qualitative longitudinal research for social policy', in Social Policy and Society, 6, 4, 529-94. [Themed Section].

Dall, T. and Danneris, S. (2019) 'Reconsidering 'what works' in welfare to work with the vulnerable unemployed: the potential of relational causality as an alternative approach', Social Policy and Society, 18, 4, 583-96.

Danneris, S. (2018) 'Ready to work (yet)? unemployment trajectories among vulnerable welfare recipients', Qualitative Social Work, 17, 3, 355-72.

Danneris, S. and Caswell, D. (2019) 'Exploring the ingredients of success: studying the trajectories of the vulnerable unemployed who have entered work or education in Denmark', Social Policy and Society, $18,4,615-29$.

Dearden, G., Good, J., Whitfield, G. and Cox, L. (2010) Credit and Debt in Low Income Families, York: Joseph Rowntree Foundation.

Donmoyer, R. (2012) 'Can qualitative researchers answer policymakers' "what works" questions?', Qualitative Inquiry, 18, 8, 662-73.

Dwyer, P. (2018) Final Findings Report: Welfare Conditionality Project, York: Department of Social Policy and Social Work, University of York. www.welfareconditionality.ac.uk

Dwyer, P. and Patrick, R. (2020) 'Little and large: methodological reflections from two qualitative longitudinal policy studies on welfare conditionality', Longitudinal and Life Course Studies, 03.07.2020.

Emmel, N., Greenhalgh, J., Manzano, A., Monaghan, M. and Dalkin, S. (eds.) (2018) Doing Realist Research, London: Sage.

Farrall, S., Hunter, B., Sharpe, G. and Calverley, A. (2014) Criminal Careers in Transition, Oxford: OUP. Ferguson, H., Warwick, L., Singh, T., Leigh, J., Beddow, L. and Disney, T. (2020) 'The nature and culture of social work with children and families in long-term casework: findings from a qualitative longitudinal study', Child and Family Social Work, 2020, 1-10.

Fleetwood, S. (2014) 'Bhaskar and critical realism', in P. Adler, P. Du Gay, G. Morgan and M. Reed (eds.), Oxford Handbook of Sociology, Social Theory and Organisation Studies, Oxford: OUP, 182-219.

Flinders, M., Wood, M. and Cunningham, M. (2016) 'The politics of co-production', Evidence and Policy, $12,2,261-79$.

Frankham, J. (2009) Partnership Research: A Review of Approaches and Challenges in Conducting Research in Partnership with Service Users, ESRC National Centre for Research Methods, eprints.ncrm.ac.uk/778/1/Frankham_May_09.pdf [accessed January 2021].

Gerrits, L. (2008) The Gentle Art of Co-Evolution (doctoral thesis), Rotterdam: Erasmus University.

Gobo, G. (2004) 'Sampling, representativeness and generalisability', in C. Seale, G. Gobo, J. Gubrium and D. Silverman (eds.), Qualitative Research Practice (Concise edition), London: Sage, 405-26.

Halford, S. and Savage, M. (2017) 'Speaking sociologically with big data: symphonic social science and the future of big data research', Sociology, 51, 6, 1132-48. 
Hammersley, M. (2008) 'Causality as conundrum: the case of qualitative inquiry', Methodological Innovations Online, 2, 3, 1-15.

Hedström, P. and Swedberg, R. (1998) 'Social mechanisms: an introductory essay', in P. Hedstrom and R. Swedberg (eds.), Social Mechanisms: An Analytical Approach to Social Theory, Cambridge: CUP, 1-31.

Hedström, P. and Ylikoski, P. (2010) 'Causal mechanisms in the social sciences', Annual Review of Sociology, 36, 49-67.

Kemmis, S. and McTaggart, R. (1988) The Action Research Planner, 3rd edn, Melbourne: Deakin University Press.

Kinsey, H. (2020) Integrating a Longitudinal Ward Placement into the Hospital Pharmacist Pre- Registration Year: A Design-based Research Approach Informing Design, Implementation and Evaluation (doctoral thesis), Norwich: University of East Anglia.

Kislov, R. (2019) 'Going native in order to make a difference? tensions of longitudinal participatory research', BMJ Open, 9, Suppl 1, 014.

Kislov, R., Wilson, P. and Boaden, R. (2017) 'The dark side of knowledge brokering', Journal of Health Services Research and Policy, 22, 2, 107-12.

Langley, A. and Tsoukas, H. (eds.) (2017) The Sage Handbook of Process Organizational Studies, London: Sage.

Langley, A., Smallman, C., Tsoukas, H. and Van de Ven, A. (2013) 'Editorial Introduction: process studies of change in organisation and management: unveiling temporality, activity and flow,' Academy of Management Journal, 56, 1, 1-13.

Le Guin, U. (2019 [1974]) The Dispossessed, London: Gollancz.

Lincoln, Y. and Guba, E. (1985) Naturalistic Inquiry, Thousand Oaks CA: Sage.

Lofland, J., Snow, D., Anderson, L. and Lofland, L. (2006) Analysing Social Settings: A Guide to Qualitative Observations and Analysis, 4th edn, Belmont Ca: Wadsworth.

Maxwell, J. (2004) 'Using qualitative methods for causal explanations', Field Methods, 16, 3, 243-64.

Maxwell, J. (2012) A Realist Approach for Qualitative Research, London: Sage.

McKenney, S. and Reeves, T. (2018) Conducting Educational Design Research, 2nd edn, London: Routledge.

Middlemiss, L., Ambrosio-Albalá, P., Emmel, N., Gillard, R., Gilbertson, J. et al (2019) 'Energy poverty and social relations: a capabilities approach', Energy Research and Social Science, 55: 227-35.

Millar, J. and Bennett, F. (2017) 'Universal credit: assumptions, contradictions and virtual reality', Social Policy and Society, 16, 2, 169-82.

Monaghan, N. and Boaz, A. (2018) 'Evidence from realist research, its influence and impact', in N. Emmel, J. Greenhalgh, A. Manzano, M. Monaghan and S. Dalkin (eds.), Doing Realist Research, London: Sage, $167-84$.

Monaghan, M. and Ingold, J. (2019) 'Policy practitioners' accounts of evidence-based policy making: the case of universal credit', Journal of Social Policy, 48, 2, 351-68.

Neale, B. (2015) 'Time and the life course: perspectives from qualitative longitudinal research', in N. Worth and I. Hardill (eds.), Researching the Life Course, Bristol: Policy Press, 25-41.

Neale, B. (2021) The Craft of Qualitative Longitudinal Research, London: Sage.

Neale, B. and Davies, L. (2015) Hard to Reach? Re-thinking Support for Young Fathers, Policy Briefing Paper no. 6, www.followingfathers.leeds.ac.uk [accessed February 2021].

Neale, B. and Morton, S. (2012) Creating Impact through Qualitative Longitudinal Research, Timescapes Methods Guides Series no. 20, www.timescapes-archive.leeds.ac.uk /publicationsandoutputs.

Nowotny, H. (2005) 'The increase in complexity and its reduction: emergent interfaces between the natural sciences, humanities and social sciences', Theory, Culture and Society, 22, 5, 15-31.

Nyström, M., Karltun, J., Keller, C. and Andersson Gäre, B. (2018) 'Collaborative and partnership research for improvement of health and social services: researchers' experiences from 20 projects', Health Research Policy and Systems, 16, 46, 1-17.

Patrick, R. (2017) For Whose Benefit? The Everyday Realities of Welfare Reform, Bristol: Policy Press.

Pawson, R. (2006) Evidence Based Policy: A Realist Perspective, London: Sage. 
Pawson, R. and Tilley, N. (1997) Realistic Evaluation, London: Sage

Penuel, W. and Gallagher, D. (2017) Creating Research-Practice Partnerships in Education, Cambridge MA: Harvard Education Press.

Pettigrew, A. (1995) 'Longitudinal field research on change: theory and practice', in G. Huber and A. Van de Ven (eds.), Longitudinal Field Research Methods, London: Sage, 91-125.

Pettigrew, A. (1997) 'What is a processual analysis?', Scandinavian Journal of Management, 13, 4, 337-48.

Pinnock, H., Kendall, M., Murray, S., Worth, A., Levack, P., Porter, M., MacNee, W. and Sheikh, A. (2011) 'Living and dying with severe chronic obstructive pulmonary disease: multi-perspective longitudinal qualitative enquiry', BMI Online, 342.

Ridge, T. and Millar, J. (2011) 'Following families: working lone mothers and their children', Social Policy and Administration, 45, 1, 85-97.

Sainsbury, R. (2012) 'How policy can ignore research: two cautionary tales', in S. Becker, A. Bryman and H. Ferguson (eds.), Understanding Research for Social Policy and Social Work, 2nd edn, Bristol: Policy Press, 373-5.

Sayer, A. (2000) Realism and Social Science, London: Sage.

Seale, C. (2018) 'Research Quality', in C. Seale (ed.), Researching Society and Culture, 4th edn, London: Sage, 567-81.

Stich, A. and Cippolone, K. (2017) 'In and through the educational 'reform churn': the illustrative power of qualitative longitudinal research', Urban Education, $6^{\text {th }}$ Feb, 1-27.

Thompson, E. P. (1978) The Poverty of Theory, London: Merlin.

Tsoukas, H. and Chia, R. (2002) 'On organisational becoming', Organisation Science, 13, 5, 567-82.

Westhorp, G. (2018) 'Understanding mechanisms in realist evaluation and research', in N. Emmel, J. Greenhaugh, A. Manzano, M. Monaghan and S. Dalkin (eds.), Doing Realist Research, London: Sage, $41-57$.

Wright, S. and Patrick, R. (2019) 'Welfare conditionality in lived experience: aggregating qualitative longitudinal research', Social Policy and Society, 18, 4, 597-613.

Wright Mills, C. (1959) The Sociological Imagination, Oxford: OUP. 